\title{
Accompagner et prévenir. Tensions éthiques dans le dépistage du cancer colorectal
}

\author{
Nicolas Lechopier*, Chloé Hamant**
}

Résumé. Cet article présente les résultats d'une enquête concernant l'éthique de la prévention. Elle a été réalisée au sein d'une recherche interventionnelle en santé des populations, mobilisant des médiateurs (navigateurs) pour corriger les inégalités de participation au dépistage organisé du cancer colorectal dans 5 départements français. Quatre tensions éthiques émergent sur le terrain : à propos de l'incertitude du test, de l'organisation des campagnes, de l'intrusion dans la vie privée et de l'universalisme proportionné. Ces tensions sont décrites à partir d'une enquête ancrée dans une approche pragmatiste, attentive aux épreuves que vivent les acteurs de la prévention, intégrant dans un même mouvement l'investigation sur les faits et la réflexion sur les valeurs.

Mots-clés : dépistage, prévention, éthique, médiation, cancer.

\footnotetext{
* Nicolas Lechopier, philosophe, Université Claude Bernard Lyon1, École normale supérieure de Lyon, EA 4148 Sciences, Société, Historicité, Éducation et Pratiques (S2HEP), 69100, Villeurbanne, France.

** Chloé Hamant, sociologue, IREPS Auvergne-Rhône-Alpes, UMR 5206 Triangle, 69007, Lyon, France.
} 


\section{Éthique et santé publique : un tournant empirique}

Le champ de l'éthique en santé publique s'est structuré depuis une vingtaine d'années en se démarquant de la bioéthique et de l'éthique clinique. Les raisons de cette démarcation sont soulignées par Dawson et Verweij (2009) : les interventions de santé publique sont orientées vers les populations et, contrairement au soin, les programmes de prévention sont la plupart du temps initiés par les professionnels de santé plutôt que par les personnes concernées ; l'autonomie individuelle mise en avant par l'éthique clinique ne peut pas être la principale pierre de touche de l'évaluation des pratiques de santé publique ; ces dernières sont mises en œuvre par les pouvoirs publics en débordant le cadre de l'institution de santé, avec une forte préoccupation pour les questions de justice et d'inégalités sociales de santé. Dans ce contexte, certains auteurs insistent sur la tension qui structure toute la réflexion éthique en santé publique, entre l'échelle individuelle et l'échelle populationnelle ou communautaire (Holland, 2007), réfléchissant aux conditions suivant lesquelles un empiétement sur la liberté des individus est acceptable (Childress et al., 2002). D'autres encore identifient des principes pour l'évaluation des interventions de santé publique (Upshur, 2002), ou des valeurs phares autour desquelles se déploie la délibération éthique (Massé, 2003).

Tous ces travaux d'éthique se soucient des spécifications de ces valeurs ou principes éthiques, de façon à ce qu'ils s'appliquent de manière pertinente à des situations concrètes. Mais ils ont été critiqués au même titre que la bioéthique pour leur tendance à traiter des questions morales à propos de situations exposées et définies par d'autres (typiquement par les médecins ou les professionnels de santé publique), et à en adopter les catégories sans les réinterroger. De leur côté, les chercheurs en sciences sociales qui font des enquêtes sur les terrains de santé publique peuvent être tentés de ne pas prendre en charge la réflexion proprement normative qui découle de leurs travaux. C'est justement cette division du travail, entre investigation des faits et réflexion sur les valeurs, qui est remise en cause dans ce que Borry et al. (2005) nomment le «tournant empirique » en éthique. La question n'est pas tant de savoir comment des principes théoriques peuvent être appliqués à des situations particulières et complexes (comme le ferait une "ingénierie éthique ») que de savoir comment articuler adéquatement recherche empirique et analyse normative. Molewijk et al. (2004) ont esquissé une typologie des approches empiriques-normatives, en prenant pour repère le choix de l'arbitre de la délibération : est-ce in fine la théorie morale ou le positionnement des acteurs qui importe ? Ou bien favorise-t-on une synergie, voire une «symbiose » (Frith, 2012) entre ces deux sources ? Dans une synthèse 
récente, Knight (2016) a repris ces typologies et montré que l'enquête de terrain pouvait, en éthique, jouer plusieurs fonctions : nourrir la délibération morale, contribuer à réviser les principes, révéler les aspects normatifs des pratiques fondées sur les sciences, ou rendre l'éthique plus sensible aux contextes. Nous présentons ici les résultats d'une enquête qui s'inscrit dans cette dernière approche, contextualisée et située, et qui cherche à rendre compte de façon circonstanciée de certaines tensions éthiques qui traversent l'expérience des acteurs dans le champ de la prévention, et qui ne sont pas forcément repérées quand on réfléchit au niveau des principes et des valeurs phares, ni même quand on s'intéresse à la façon dont les principes s'appliquent à des cas particuliers.

\section{Accompagner le dépistage organisé du cancer colorectal}

Le dépistage organisé (DO) est un programme de santé publique consistant à repérer, de façon précoce, chez des individus sans symptômes, et dans l'ensemble d'un territoire, des cas de maladie, dans le but de mieux les prendre en charge. Cette approche de la prévention, dirigée à la fois vers la population générale et sur une pathologie spécifique, apparaît comme l'inverse des approches de prévention sélective ou ciblée, visant un sous-groupe à risque particulier. De plus, le dépistage organisé consiste, non pas à travailler sur les facteurs permettant d'éviter ou de faire reculer la survenue d'une maladie, mais bien à recruter des personnes qui n'ont pas de symptômes ni de facteurs de risque particuliers pour leur faire réaliser des examens médicaux. Comme cela a été bien montré il y a quelques années dans un numéro de Sociology of Health and Illness, le dépistage constitue un objet sociopolitique tout à fait particulier (Armstrong et Eborall, 2012). Dans le présent article, nous montrons que le $\mathrm{DO}$ est un lieu où se révèlent certaines tensions éthiques particulières, mais nous suggérons que ces tensions traversent, probablement, le champ de la santé publique dans son entier.

Pour penser ces tensions, il faut considérer les pratiques de santé publique et les normes éthiques comme étant en interaction. Dans le cas du DO, elles appartiennent à la même histoire : c'est au début des années 1970 qu'ont été mises en place les premières campagnes de DO, et c'est précisément au même moment qu'a été formulée la série de critères permettant d'en évaluer l'opportunité (Wilson et Jungner, 1970). Ces critères sont : importance (gravité, fréquence) de la pathologie dépistée, efficacité et disponibilité d'un traitement, existence de connaissances suffisantes disponibles à propos de l'histoire naturelle de la maladie, exis- 
tence d'un plan d'action coordonné, proportionnalité entre les coûts de diagnostic et de traitement par rapport au coût global des soins médicaux, acceptabilité du dépistage pour la population concernée. Selon certains auteurs, ces critères forment un consensus qui reste valable aujourd'hui pour juger de l'opportunité et fonder une éthique du dépistage (Juth et Munthe, 2012). Mais, selon nous, trois dimensions manquent.

D'abord, les incertitudes des connaissances épidémiologiques à propos du DO restent grandes, et sont d'autant plus gênantes que les dépistages concernent des millions de personnes (Skrabanek, 1990). Cela étant, dans la présente enquête, nous nous intéressons au dépistage organisé du cancer colorectal (DOCC), qui est l'un de ceux qui soulèvent le moins de controverses, notamment en termes de rapport bénéfice/risque (1) (nous y reviendrons). Ensuite, le consensus de 1970 ne mentionne pas clairement les préférences de la population ou la liberté de choix des individus, si ce n'est à travers le prisme de l'acceptabilité. Or, on reconnaît depuis aux personnes ciblées un droit à être informées et reconnues dans leur capacité à juger, à l'aune de leurs préférences et de leurs propres valeurs, de l'intérêt d'y participer ou non (Barratt et al., 2002 ; Collectif, 2016). Enfin, à ces critères manque l'enjeu des inégalités sociales de santé qui sont creusées, au lieu d'être réduites, par les politiques de prévention. Une synthèse portant spécifiquement sur le DOCC souligne que, dans la vingtaine de pays où ce dépistage est organisé, partout les inégalités de participation tendent à refléter le gradient social de santé (Potter, 2013). Incertitudes, droit individuels et inégalités sont trois enjeux ouverts, qui ne sont pas réglés et qui, selon nous, sont constitutifs de différentes tensions proprement éthiques. Comment ces enjeux s'articulent-ils sur le terrain?

(1) Conduit en deux temps, avec un autotest de repérage de sang occulte dans les selles, suivi en cas de résultat positif d'un examen coloscopique, le dépistage de ce cancer a un rapport bénéfice/risque moins discutable que les dépistages d'autres cancers (Hill, 2013). D'après la synthèse Cochrane, pour 1000 personnes entre 50 et 74 ans effectuant le test tous les 2 ans pendant 15 ans, 7 personnes mourront quand même de ce cancer, contre 8 dans le groupe de personnes n'effectuant pas le test, tandis que moins d'une personne sur mille a des complications médicales importantes dues à la coloscopie (Holme et al., 2013) et ce, même si la coloscopie comporte des désagréments fréquents et non négligeables (Ponchon, 2009). Au final, le dépistage organisé contribue à diminuer la mortalité attribuable au cancer colorectal dans la population. 


\section{Une éthique embarquée}

Une recherche interventionnelle en santé des populations a été réalisée entre 2012 et 2015, financée par l'Inca et la Ligue contre le cancer. Son but était d'étudier les conditions d'application, dans le contexte français, d'un programme de médiation santé inspiré du Patient Navigation Program américain, pour réduire les inégalités dans les campagnes de dépistage du cancer colorectal (Allary et al., 2016). Le «navigateur » n'est pas d'abord professionnel de santé ou du social, mais un pair qui agit en principe de façon transversale par rapport aux professionnels du secteur, partageant avec la population cible des caractéristiques communes. Les médiateurs-navigateurs avaient une mission de 18 mois dont le but était de faire augmenter le taux de participation au DOCC parmi les populations vivant dans des zones IRIS socio-économiquement défavorisées, tirées au sort au sein de 5 départements français. La recherche interventionnelle visait à savoir si l'intervention du navigateur permettait aux personnes issues des zones concernées de mieux comprendre l'intérêt du dépistage et de réaliser le test, par comparaison à des zones voisines et semblables en termes socio-économiques, dans lesquelles un navigateur n'était pas présent.

Dans une enquête en « éthique embarquée » (Lechopier, 2015), nous nous sommes proposés de décrire et documenter les enjeux éthiques de facto soulevés par les acteurs, en mobilisant des ressources philosophiques et de sciences sociales, et en ouvrant des espaces de réflexivité avec navigateurs, habitants, chercheurs, porteurs du projet, etc. Outre des observations lors des journées de formation des navigateurs et des entretiens semi-directifs auprès de grands témoins du champ du DOCC, l'une des originalités de notre étude est d'avoir réalisé des entretiens collectifs sous la forme de débats éthiques. L'analyse de ce matériau a suivi deux axes. L'axe synchronique consistait à délimiter l'espace du sujet traité et à comprendre l'organisation des registres d'argumentation, des objets, des acteurs, etc. L'axe diachronique consistait à rendre compte des ajustements et de la constitution de nouveaux arrangements au fur et à mesure du déploiement de l'intervention. Ces échanges avec et entre les chercheurs et les navigateurs au début et à l'issue de l'intervention, ainsi qu'avec des habitantes d'une des zones d'étude, ont permis de jalonner la dynamique des expériences morales sur le terrain et de décrire les rétroactions entre les valeurs et les pratiques. Dans cette enquête d'inspiration pragmatiste, faits et valeurs ne se trouvent pas séparés mais entremêlés (Putnam, 2004) et c'est dans des « épreuves » ou des situations problématiques (Dewey, 2011) que l'on a l'occasion de saisir leurs dynamiques et leurs réajustements. Les résultats sont présentés autour des quatre tensions qui ont été dégagées au cours de cette enquête. 


\section{Entre accompagnement et incertitudes}

La première tension que nous avons explorée se déploie entre, d'une part, le travail sur les barrières à la participation au DO et, d'autre part, la prise en compte des incertitudes liées à la campagne de dépistage.

Précisons d'abord que l'action des navigateurs consiste, par un travail d'accompagnement spécifique, à augmenter la participation des personnes qui vivent dans des zones où, en moyenne, on se dépiste moins. Il s'agit de lever les barrières qui empêchent les gens de participer à cette campagne. Sans que cela soit forcément clairement précisé par les porteurs du projet, le travail de navigateur suppose de faire une distinction entre les barrières structurelles (revenus, précarité, etc.) sur lesquelles il ne peut agir directement, et les barrières qui sont, elles, actionnables. Ce sont celles qui affectent la compréhension et l'usage du dispositif, l'accès à l'information ou aux soins, ou encore les "représentations », à propos desquelles un dialogue à long terme peut s'amorcer.

La particularité du test de dépistage du cancer colorectal est d'être un autotest visant à détecter le sang occulte dans les selles. Il sera, dans la suite de ce texte, question du test Hemoccult, qui a depuis été remplacé par un test immunologique (2). Aucun autotest n'est réductible à un procédé d'analyse ou à une réaction chimique. Sa validité dépend d'une succession de petits gestes que les personnes doivent exécuter ellesmêmes : il fallait récupérer le test Hemoccult auprès du médecin (ou le recevoir par la poste), comprendre comment on l'utilise, mettre en place une stratégie pour pouvoir accéder à ses selles, en prélever différents échantillons à différentes occasions les déposer sur la zone autocollante de l'enveloppe fournie, envoyer le tout par la poste, récupérer les résultats et en tenir compte, en prenant rendez-vous pour une coloscopie en cas de résultat positif. C'est dans toute cette série de gestes - que les navigateurs nomment «faire sa prévention» - qu'il faut apprendre à naviguer.

(2) En France, le test Hemoccult utilisant le gaïac pour détecter des traces de sang dans les selles et déployé par la campagne de DOCC depuis sa généralisation en 2009 a été remplacé dans le courant de l'année 2015 par un test immunologique intitulé «OC sensor ». Il s'agit encore d'un test à effectuer soi-même, mais qui ne nécessite qu'un seul prélèvement ; il est donc moins contraignant que le test Hemoccult. Contrairement à ce dernier, il ne réagit pas à l'hémoglobine animale issue de l'alimentation. En revanche, il ne distingue pas mieux l'origine des saignements. Il entraîne donc aussi des faux positifs. Ce nouveau test, plus sensible, pourrait multiplier par deux le nombre de coloscopies réalisées au titre du dépistage. 
Les navigateurs se sont donc centrés sur la bonne utilisation du test Hemoccult. Mais il reste que celui-ci n'est pas forcément un bon test. Il est peu spécifique, puisqu'il ne peut pas différencier si le saignement provient d'un cancer du côlon ou d'une source bénigne, et il réagit à certains aliments. Il est également peu sensible : "Si vous avez effectivement un cancer, vous avez une chance sur deux pour que le test Hemoccult ne détecte rien » (Chercheur). L'argumentaire utilisé par l'un des navigateurs reprend les mêmes informations, en les présentant sous leur aspect plus positif : " $94 \%$ des cancers de l'intestin apparaissent après 50 ans et 1800 personnes ont été sauvées grâce au dépistage [1 200 dans une autre version]. Deux à $3 \%$ seulement des tests sont positifs. Le test trouve $d u$ sang invisible à l'œil. Alors un examen visuel du colon est fait. C'est la coloscopie! 1 fois sur 10 c'est un cancer, 3 fois sur 10 c'est un polype qui peut se transformer en cancer » (Navigateur, dépliant de présentation).

Cette présentation qui combine pourcentages et proportions permettait à la personne contactée d'apprécier l'importance du problème du cancer colorectal (même si on ne sait pas combien il y a de cas de cancers), les apports du dépistage organisé (mais sans parler des inconvénients des coloscopies inutiles), et les différentes étapes et les bonnes chances de ressortir indemne de ce processus (3). En pratique, un résultat négatif au test doit être considéré comme peu informatif ; en revanche un résultat positif (qui reste peu fréquent) est présenté comme une information potentiellement très utile, même si le plus probable est que ce soit un faux positif qui occasionne une coloscopie inutile. En bref, le test Hemoccult n'était pas très sûr ni très puissant, mais sans danger : " c'est facile et ça peut rapporter gros » (Médecin). Un navigateur proposait de s'en tenir à ce point de vue pragmatique et de ne pas tergiverser. "On essaie de leur dire que le test Hemoccult peut leur sauver la vie. Certes, il y a des puristes, des scientifiques qui disent qu'il n'est pas bon. Mais c'est tout ce qu'on a. Et si on ne l'avait pas, il n'y aurait rien » (Navigateur).

Il s'agissait donc de gagner la confiance des habitants et des autres professionnels, de sensibiliser les personnes qui étaient dans la « cible » et de les faire se sentir concernées par le dispositif de dépistage. Mais les

(3) Les modalités de présentation de données, sous forme de quantités, de fréquences ou de risques relatifs, déterminent pour une bonne part la façon dont elles sont comprises et reçues (Gigerenzer, 2008). Selon la présentation retenue, on peut avoir l'impression que le même test est extrêmement efficace ou bien au contraire extrêmement peu fiable. 
habitants et les partenaires des navigateurs ont entendu parler du remplacement d'Hemoccult par le test immunologique, et il est arrivé que certains disent qu'on leur avait jusque-là fait la promotion d'un test pas si efficace et pas si fiable que ça. Un des navigateurs raconte qu'il a dû batailler pour arriver à reconquérir la confiance des habitants à propos du test Hemoccult, dont les imperfections n'étaient pas inclues dans le modèle explicatif du problème à résoudre par le navigateur. Le modèle des barrières repose sur le présupposé selon lequel, sans ces barrières, la personne effectuerait à coup sûr le test. Or, ce présupposé se trouve remis en question lorsque se font jour des difficultés à expliciter et assumer les incertitudes et les imperfections du programme et des technologies sur lequel il repose.

On retrouve là un paradoxe désormais bien connu : les pouvoirs publics ont tendance à craindre qu'informer soigneusement les personnes de l'efficacité réelle (limitée) des tests ne conduise à une baisse de participation ; mais ce faisant, ils courent le risque qu'une présentation artificiellement favorable, ne tenant pas compte des retours d'expérience des personnes ou des savoirs qui circulent à propos des technologies impliquées, ne conduise à une perte de confiance pouvant elle aussi faire baisser les taux de participation. L'un de nos interviewés pointe là une situation de conflit d'intérêt qui traverse le champ de la prévention médicalisée : "[les autorités de santé] sont partie prenante de l'évaluation du résultat en termes de couverture de leur programme et elles mettent beaucoup d'argent; donc elles veulent du résultat et elles veulent pas prendre de risque ... elles se disent : "si j'informe sur les risques, je vais faire plonger ma moyenne" " (Chercheur). Les travaux de Steckelberg et al. (2011) ont pourtant montré, à propos du DOCC, que l'information soigneuse à propos de l'efficacité réelle du dépistage du cancer colorectal n'a guère d'impact positif ou négatif sur les taux de participation, même si l'on peut considérer qu'elle permet une participation de meilleure qualité - une participation plus « consciente ». Une information complète permet aux personnes estimant qu'elles feraient bien de faire le test, de le faire de façon lucide et aux autres de savoir ce qu'elles acceptent de risquer en ne le faisant pas. Le taux de participation ne change pas. $\mathrm{Ce}$ qui change, c'est qui participe et pour quelles raisons.

On est là au cœur d'une première tension qui traverse non seulement le dépistage organisé mais semble-t-il aussi toute la prévention en santé publique. L'intervention de navigateurs vise à augmenter la participation là où elle est faible. Pour cela, il faut non seulement apporter toutes les médiations nécessaires, mais aussi une technologie en laquelle on peut avoir confiance. Or, cette confiance est rendue difficile à cause des limitations techniques intrinsèques du test, mais aussi à cause de la difficulté 
à le présenter avec toutes ses limites et son avantage individuel ténu. La prévention de santé publique est prise ici entre deux polarités : elle se déploie comme un processus industriel visant une large couverture de la population et se présente en même temps comme un milieu accueillant les incertitudes et reconnaissant les droits des participants.

\section{Entre catégories et singularité}

La seconde tension que nous avons décelée au cours de cette enquête est celle qui naît du décalage entre les catégories de la santé publique et celles qui organisent l'expérience des personnes concernées. Les programmes de prévention sont organisés en secteurs qui reflètent euxmêmes un découpage des maladies en pathologies et un découpage d'une sous-partie de la population en un public cible. Les navigateurs, en tant qu'acteurs de santé publique, relaient ce projet de santé publique. Leur travail est cadré sur une pathologie, un organe (intestin, rectum) et est orienté par la finalité étroite de faire réaliser à un maximum de personnes un test de dépistage. Leur mission est d'intervenir et d'accompagner les individus dans les zones qui participent moins au dépistage. Mais, en ayant pour rôle spécifique de prendre les individus là où ils en sont et de les accompagner dans la prévention (Parker et al., 2010), ils ne suivent pas (et ne peuvent pas suivre) les divisions thématiques, sectorielles, institutionnelles de la santé publique qu'ils sont pourtant obligés de prendre en compte dans leur action. D'où cette seconde tension, entre une approche descendante et ciblée de la prévention de santé publique et une approche holiste revendiquée en santé communautaire dans une optique de promotion de la santé.

Un premier moteur de cette tension découle des logiques d'acteurs dans le tissu local, telles qu'explorées dans une phase initiale du projet par Pommier (2015) puis par Buthion et al. (2015). Le champ de la prévention y est découpé en différents programmes et traversé par des frontières de territoires, comme en a fait l'expérience une navigatrice cherchant à nouer des contacts dans sa zone d'activité : "En CCAS, et ça m'a vraiment choquée, la dame qui s'occupe [...] des projets de prévention, vous vous imaginez quand j'ai pris rendez-vous ? [...] elle m'a dit: "oui oui, ça concerne quoi ?", je lui ai dit: "bon, c'est le dépistage du cancer colorectal, donc c'est un projet, c'est pour faciliter l'accès au...", alors elle m'a dit : "ah, je regrette, Madame, nous on fait de la prévention du sein" » (Navigatrice). 
Les différentes campagnes de dépistages sont présentées comme indépendantes et parallèles, alors qu'elles se croisent dans les lieux où elles sont relayées et, bien sûr, chez les populations-cibles. Le déploiement en silos des campagnes de santé publique heurte aussi la logique spécifique que l'on a décrite plus haut, qui définit l'action du navigateur comme chargé d'un accompagnement individuel. Le navigateur a en quelque sorte pour fonction, en partant de là où en sont les gens, de modifier la hiérarchie de leurs priorités en valorisant celle du dépistage et de les aider à l'accomplir effectivement. Bien entendu, s'occuper de sensibiliser une personne sur ce dépistage en particulier n'interdit nullement d'évoquer d'autres actions de prévention ou de soins primaires pour des maladies comme le diabète. Cela n'empêche pas plus d'aborder les problématiques économiques, familiales, de logement, etc., que connaissent ces personnes. Mais l'intervention reste focalisée et pensée à travers le prisme de l'intervention de santé publique à visée de dépistage d'une maladie en particulier.

Cette situation est-elle tenable ? Elle met à l'épreuve les navigateurs qui en discutent longuement lors d'un débat éthique, notamment à propos de l'approche des personnes en situation de pauvreté et de précarité : «J'ai rencontré des personnes qui avaient très peu de revenus [... ] l'une d'elle était au courant donc du test et il m'a dit, "non tant que j'ai pas réglé mon problème, de toutes façons, je peux pas m'occuper de ma santé" ... Je lui dis, "écoutez Monsieur, c'est pas grave vous vous occuperez de vos problèmes financiers, le test, ça peut attendre..." Et là à la limite, c'est nous qui avons honte! Le monsieur, il n'est déjà pas bien parce qu'il a des problèmes financiers et on est là, on lui parle d'un problème de santé... bon c'est bien, c'est la santé, mais après voilà, on est humain et on se dit "peut-être que je vais déranger"? Ou alors je lui ai rendu un service en lui parlant de ça? » (Navigateur).

Alors qu'au cours de la formation des navigateurs, la honte et autres expériences morales avaient été pointées comme autant de barrières potentielles à lever, c'est en ces mêmes termes que cette intervenante décrit son expérience. Comment rendre service à une personne, sachant qu'il s'agit à la fois de tenir compte d'une perspective individuelle et de considérer la personne dans son écologie familiale et sociale ? Comment faire lorsque la prévention d'une maladie simplement possible n'entre pas dans l'horizon de préoccupation d'une personne qui a d'autres problèmes plus urgents à résoudre?

Tout cela pourrait se comprendre comme un dilemme à traiter à travers les modalités traditionnelles de la délibération éthique. Dans une optique utilitariste, on se posera la question de savoir si les bénéfices attendus des programmes de prévention (en l'occurrence, par recours au 
dépistage du cancer colorectal) l'emportent sur les contraintes que ces programmes font porter sur les individus, notamment ici en termes d'inquiétude supplémentaire, de changement de priorités, de frustrations, etc. Dans une optique plutôt déontologique, on se penchera plutôt sur l'arbitrage à faire entre le respect des choix éclairés des personnes et le devoir pour chacun de faire ce qu'il convient pour se maintenir en bonne santé. Arbitrage, autrement dit, entre une logique du choix et l'exigence faite aux individus de "se conserver soi-même » que l'on trouve chez Locke (comme application de la loi naturelle) ou chez Kant (comme devoir envers soi-même). La réflexion éthique peut aussi réinterroger ces dilemmes en redéfinissant la notion même de santé qui, entendue plus largement comme "bien-être », ne saurait s'imposer aux individus à travers une injonction déterminée, mais devrait bien plutôt être pensée comme une qualité ou une vertu à cultiver. Ici, pour éviter de raisonner seulement à partir de principes généraux, nous choisissons plutôt de poser cette question en tenant compte du contexte dans lequel se déroulent les interventions de santé publique, de qui en sont les acteurs, ainsi que de la perspective des personnes concernées et « ciblées ». Or, la question éthique qui découle de l'expérience des acteurs se révèle dans un conflit, " conflit entre ce besoin, cette demande de résultats, qui est quand même la raison pour laquelle on est là, en partie, et le fait de vouloir faire une relation humaine, où on fait de la prévention, pour le bien-être des gens » (Navigateur).

La question de fond qui se pose ici est d'ordre politique. Elle touche à l'articulation entre un programme et des personnalités singulières. Bon nombre de programmes de prévention ont été ces dernières années analysés à partir du prisme du «paternalisme libéral ». Cette approche articule le principe selon lequel l'État ou les autorités de santé publique savent mieux ce qu'il faut faire pour prendre soin de sa santé, et le principe plutôt libéral selon lequel chaque personne, au carrefour de différentes appartenances, est libre de choisir et de définir ce qui, pour lui ou elle, importe le plus - tant qu'autrui n'est pas exposé à des préjudices du fait de ce choix. Mais cette synthèse de paternalisme et de libéralisme ne permet pas d'éclairer l'expérience morale des personnes qui se situent à l'endroit précis où s'accomplit le saut individu/population. Comment et jusqu'à quel point un programme de santé publique déployé à l'échelle populationnelle peut-il faire place à la singularité individuelle, à la personnalité des sujets qu'il cible? Alors qu'en clinique, la notion d'art médical et le registre du colloque singulier permettent de surmonter la tension entre l'échelle du singulier et celle du collectif, il n'existe pas encore une telle articulation dans le champ de la prévention. C'est pourtant cette médiation-là dont sont chargés les navigateurs, les « médiateurs-pairs ». 
Alors que la santé publique se déploie, là encore, sur un mode industriel (objectifs, cibles, processus, évaluation, qualité, rendement, etc.), ce n'est jamais un «individu lambda » ou un membre de la société défini par ses caractéristiques socioprofessionnelles qui prend soin de soi, mais bien une personne, irréductible à des catégories génériques. Les navigateurs sont là encore à un point instable, pris entre deux pôles : ils se présentent comme agents de déploiement d'un programme de dépistage, mais ils conçoivent leur démarche dans un registre de l'accompagnement individuel et personnel - un registre « humain ».

\section{Entre respect et efficacité}

La troisième tension prolonge directement la précédente mais fait l'objet d'un traitement spécifique et explicite à l'occasion d'épreuves multiples. Elle concerne l'enjeu proprement éthique du respect des personnes et la question du caractère intrusif des campagnes de prévention de santé publique. C'est cette question-là qui aura été la plus présente au cours des débats éthiques. Dans la littérature contemporaine en éthique de la santé publique, l'angle est celui de la juste mesure de l'empiétement, au nom de la santé publique, sur le domaine privé de l'individu pris dans son acceptation libérale (Holland, 2007). Quel degré d'intrusion dans la vie des gens (ou quel degré d'interférence avec leurs trajectoires de vie) est acceptable au regard des bénéfices (collectifs et individuels) potentiellement apportés par une intervention préventive ? Dans la perspective de l'éthique embarquée adoptée ici, nous nous sommes intéressés à l'émergence de cet enjeu, en nous posant la question des expériences et des explicitations auxquelles il donnait lieu.

La question de savoir jusqu'où il était possible d'aller, pour un navigateur, a émergé au sein d'un questionnement plus général qui consistait à articuler deux exigences : d'une part, faire attention aux personnes et individualiser le contact avec elles et, d'autre part, contacter le plus grand nombre de personnes dans la population cible et réussir à faire augmenter le taux de participation dans la zone d'intervention. Le travail de frontières qui a été réalisé par les acteurs de la recherche a précisément consisté à trouver une façon d'assumer la tension entre ces deux registres.

La justesse de la démarche du navigateur et le tracé des limites du respect des personnes ont été progressivement dessinés : la première étape remonte à la formation initiale qui avait été proposée aux navigateurs peu après leur recrutement. Cette formation plutôt intensive, étalée sur une semaine, incluait des éléments de formation, de techniques de communi- 
cation, de logistique et de déontologie. Le module " éthique » fut confié à une psychologue du travail qui délivra une formation orientée autour d'objectifs explicites : «respect de la confidentialité, attitude d'empathie, ne pas vendre le test, absence de jugement de valeur » (Formateur). Lors des travaux en petits groupes, ce sont les aspects de communication et de posture qui ont été discutés : être avenant, accessible, neutre, avec une image simple et zen ( «ni costume-cravate ni piercing »). Il était question de confiance à créer avec les autres acteurs, d'une humilité à conserver («on n'est pas médecin », dit à ce propos un navigateur). Il s'agissait d'apporter aux personnes de la population cible des informations adaptées, d'écouter et de donner des arguments, et non de chercher à convaincre ni à persuader. Le cadre posé était celui de la promotion de la santé et du respect de la liberté de choix de la personne, à qui il est donné des informations adaptées et les moyens de faire un dépistage, puisque c'est elle qui décide de faire le test.

Après quelques semaines de terrain pendant lesquelles les navigateurs durent essayer de se faire une place dans leur zone d'action, à l'occasion d'une première réunion d'échanges de pratiques proposée par l'équipe de pilotage du projet, un premier débat éthique fut organisé. La discussion se cristallisa sur le maintien de cette limite qui avait pourtant été tracée lors de la formation : "le navigateur est là pour informer mais pas pour convaincre ». Cette frontière vacillait déjà puisqu' on demandait également aux navigateurs, non pas de présenter de façon neutre une information, mais de se montrer favorable au dépistage et d'essayer d'en faire parler le plus possible autour d'eux. Certains navigateurs estimaient d'ailleurs que leur mission était tout simplement de "persuader les personnes rencontrées de se faire dépister », tandis que d'autres estimaient qu'il fallait surtout éviter de mettre la pression ou d'être intrusif, et de se contenter d'être disponible pour qui s'intéresse au dépistage. « $L a$ prévention c'est toujours des propositions.... on n'est pas dans l'injonction, il y a pas une note, on va pas faire un devoir [...] aujourd'hui les gens on leur met à disposition des informations grâce à une proximité hein qui peut à un moment ou un autre les faire réagir, les amener par eux-mêmes à prendre des décisions concernant l'importance de leur santé » (Navigateur).

Dans ce premier débat, la question était surtout de savoir à partir de quand le navigateur qui fait la promotion du dépistage «tombe dans le démarchage ». L'un des membres de l'équipe de recherche était intervenu dans le débat et provoqua les navigateurs en affirmant qu'il ne fallait pas avoir peur de penser en termes de chiffres et de ventes, comme dans le domaine commercial. Cet intervenant évoqua même l'idée que des primes de résultats pourraient être distribuées aux navigateurs qui feraient de 
bons chiffres. Les navigateurs récusèrent en bloc cette assimilation de leur rôle à celui de vendeur. Ils se posèrent cependant la question suivante : en admettant que le test soit indubitablement bon pour la santé, comment à la fois le vendre (au sens figuré : en faire la promotion), sans réduire la prévention à un produit commercial, sans assimiler le navigateur à un VRP ? Les navigateurs trouvèrent un arrangement qui consistait à dire que la juste posture consistait à aller vers les personnes et à les accompagner dans leur propre choix à propos du dépistage, sans pour autant chercher à les convaincre de quoi que ce soit (sous peine de tomber dans le registre commercial).

Ce débat aura des conséquences pour la suite car se construira progressivement un jugement de valeur à propos des différentes approches que mettent en œuvre les navigateurs, qui seront bientôt classés en deux catégories, les « proactifs » et les « attentistes », ces derniers s'exposant au risque de toucher peu de monde et de ne pas réussir à faire changer la participation au DOCC. D'ailleurs, la crainte d'un échec conduisit l'équipe de pilotage du projet à faire un net recadrage (que les navigateurs vécurent assez mal) en rappelant que leur but était clairement d'augmenter la participation au DOCC. Et que s'il ne s'agit pas bien sûr d'empiéter sur la liberté des personnes de ne pas y participer, il était nécessaire de faire le maximum pour accomplir cet objectif - et donc ne pas rester dans une posture « attentiste ».

Les navigateurs furent tenus de composer avec ce nouveau coup de barre. Pris dans une tension entre le respect des personnes («ne pas être intrusif ») et la nécessité de faire augmenter un taux de participation ( faire $d u$ chiffre »), les navigateurs établirent lors du second débat éthique un nouvel arrangement. S'il fallait certes ramener des résultats et augmenter les dépistages, les navigateurs affirmaient cependant que leur propre éthique était d'être authentiques et sincères avec les populations, d'être effectivement utiles, sans les instrumentaliser, sans en faire des moyens de leurs propres fins. La position du navigateur, certes polarisée par la promotion du dépistage, s'avérait surtout caractérisée par l'honnêteté et le désintéressement, ces vertus fournissant le repère éthique du métier, par-delà l'exigence d'efficacité faite aux individus.

Cet argument de la sincérité généreuse semble avoir puisé dans l'expérience de certains navigateurs en tant que bénévoles de la Ligue contre le cancer, ou du travail caritatif, ou encore dans leur engagement dans des communautés religieuses. Ce registre humanitaire ou du bénévolat admet une certaine asymétrie entre celui qui donne (sincèrement) et celui qui reçoit (parce qu'il conçoit que c'est son bien). Toute notion d'échange ou de condition particulière était explicitement écartée par les navigateurs. «C'est un système pour orienter les personnes. Imagine quelqu'un, dans 
la rue, qui est perdu. Je vais pas lui dire "je vais te montrer le chemin, mais à condition que...” » (Navigateur).

Ce registre du don désintéressé avait l'avantage de permettre de se démarquer clairement du registre commercial qui présupposait que celui qui vend y prend un intérêt personnel ou individuel. Il fallait donc aux navigateurs être informatif et convainquant, faire du chiffre sans être vendeur tout en restant sincère. La marge de manœuvre sous cet ensemble de contraintes - voire d'injonctions contradictoires - était étroite, et l'on a observé un déplacement en cours de route à propos de la juste posture à l'égard des personnes. La tension entre efficacité de l'intervention en population et respect de chaque personne a trouvé là une ébauche de résolution dans une éthique de la vertu, valorisant le registre asymétrique de l'aide.

\section{Entre universalisme et expériences situées}

Une quatrième et dernière tension est apparue dans notre enquête. Elle concerne cette fois la façon dont la lutte contre les inégalités de santé, qui est l'un des axes structurant des politiques de santé publique contemporaines, se traduit dans des pratiques de prévention mobilisant des médiateurs-pairs. Si les inégalités de santé s'expliquent par des déterminants sociaux (Marmot, 2005), elles sont aussi dépendantes de facteurs propres aux politiques de santé. On sait que les programmes de dépistage organisé ont tendance à profiter davantage aux classes qui sont déjà en meilleure santé et donc à creuser les inégalités sociales de santé. Le consensus actuel engage à mettre en place des politiques spécifiques pour corriger ou pallier ces inégalités. "L'universalisme proportionné » est l'appellation désormais consacrée pour désigner la part d'adaptation aux particularités que l'on adjoint aux politiques visant la population générale (Lang, 2014). Cela consiste à tenir compte des inégalités de départ et à viser une égalité non pas arithmétique mais géométrique, orientée par une conception de la justice comme équité. Le dispositif de navigateurs vient compléter la campagne de dépistage organisé pour toute la population en visant à améliorer le dispositif pour les catégories qui participent moins (ne se sentent pas concernées, sont les plus éloignées de la prévention, etc.).

La façon dont les navigateurs réalisent cette fonction correctrice révèle les tensions qui traversent cette ambition. Les inégalités sociales de santé se repèrent à travers des découpages socio-spatiaux (zones IRIS) qui servent aussi de ciblage pour les interventions de santé publique. Mais les 
navigateurs travaillent en réalité sur un territoire dont les espaces et les circulations ne coïncident pas nécessairement avec ces découpages-là. Les habitants eux-mêmes y échappent. "Je ne fais pas le tri, même au niveau des zones, je ne demande pas : "excusez-moi, vous êtes de quelle zone ?" "(Navigateur). De même, alors que les inégalités de santé se caractérisent par un gradient social de santé dans toute la population, la définition de la population pour laquelle il faut prévoir un rattrapage ou un surcroît d'accès est nécessairement arbitraire. Une première difficulté pour les navigateurs consiste donc à concevoir leur action en tenant compte de la population cible (de 50 à 74 ans, habitant une zone tirée au sort parmi celles qui montrent une moindre participation aux campagnes) mais aussi des individus particuliers avec qui ils établissent une relation particulière, et qui ne se conçoivent pas nécessairement à travers le prisme de ces catégories-là. Pour toutes ces raisons, il est difficile pour les navigateurs du projet d'expliciter l'optique corrective de l'intervention.

La seconde difficulté, directement liée à la précédente, tient à ce que l'on fait porter sur le navigateur, acteur minoritaire et peu doté en moyens d'action, la responsabilité de corriger des inégalités sociales de santé. La médiation santé et les navigateurs pour le dépistage représentent, selon une métaphore développée par un chercheur, qu'une " rustine sur une chambre à air percée ». Loin d'être dépréciative, cette analogie de la médiation santé avec la rustine est plutôt juste. En effet, de même que la rustine n'est pas une technologie complexe mais simple et robuste afin de rétablir (au moins temporairement) le bon fonctionnement d'une roue, de même la navigation est une low technology pour produire des moyens simples et contextuellement pertinents pour favoriser l'accès à une ressource de santé. On attend ainsi des navigateurs qu'ils développent des astuces simples, efficaces, ponctuelles et contextualisées pour permettre la participation au DOCC. On attend qu'ils mettent au point une approche pragmatique, adéquate au terrain et aux populations dont ils sont familiers, en bref qu'ils soient des innovateurs en matière de correctifs aux inégalités de santé. Cette attente s'est traduite par la formule fréquemment utilisée : "l'invention de son propre métier », les navigateurs devant établir leurs propres conditions de travail (trouver un local, construire leur matériel de communication, se faire un réseau, etc.). Peu à peu, devant les difficultés concrètes que rencontraient les navigateurs pour commencer à travailler et obtenir un début de reconnaissance, ce registre de l'invention d'un métier fut abandonné, au profit d'une focalisation sur la façon d'aborder les gens et produire de l'accompagnement. Les chercheurs souhaitaient apprendre de la part de ces navigateurs « autochtones » à mettre en place des programmes de santé publique qui tiennent mieux compte des populations les plus éloignées de la prévention. Cependant, ces navigateurs étaient 
également pris dans différentes contraintes (approches top-down et sectorialisées de la santé publique, critères étroits de performance, etc.) qui rendaient difficile ce travail d'innovation.

Troisièmement, des réticences se sont exprimées à propos du registre des inégalités sociales comme cadre conceptuel et éthique. La notion démographique et épidémiologique d'inégalités sociales de santé pointe implicitement les populations défavorisées, avec tout ce que cette catégorie a de stigmatisant pour ceux et celles qui y sont inclus-es. Il y a une forme de violence symbolique à désigner, sur la base d'informations statistiques, un lieu de vie comme étant celui d'une " population défavorisée ». Un habitant du département de la Loire réagit vivement à cette étiquette : "Pourquoi "défavorisés" !? Est-ce qu'on vit dans la misère? Est-ce qu'on n'a pas d'électricité !? Est-ce qu'on n'a pas de chauffage!? On n'est pas défavorisés!» (cité dans Pommier, 2015 : 96). Et les navigateurs eux-mêmes éprouvent des difficultés à s'emparer de cette notion statistique et démographique. "Cette histoire d'inégalités est un peu embêtante. Je ne peux pas dire que je suis venu parler du dépistage spécialement parce qu'il s'agit d'une zone défavorisée » (Navigateur). Cet écueil est lié à une certaine conception des inégalités en santé publique, davantage fondée sur des savoirs démographiques et épidémiologiques que sociologiques et expérientiels. Cette conception renvoie à des étiquettes et à des catégories statistiques - parfaitement légitimes et utiles dans le cadre d'un plaidoyer basé sur les travaux d'épidémiologie sociale - mais qui, comme le remarque aussi Mazet (2013) ne prennent pas en compte les savoirs d'expérience et les points de vue des personnes concernées par les phénomènes de non-recours ou de discrimination.

Une quatrième et dernière difficulté concentre les précédentes. Il nous semble que cette recherche interventionnelle mobilisant des médiateurs navigateurs pairs pour corriger des inégalités sociales de santé a globalement souffert d'une conception trop vague de la notion de pair. Pourtant cette notion fait explicitement partie des sources étasuniennes du patient-navigator, dont le projet français s'est inspiré. Plusieurs qualificatifs ont circulé pour rendre raison de la proximité attendue des navigateurs avec les populations cibles : «familiarité », «être du même quartier », être « issu de la même population » («immigrée » ou « maghrébine »), ne pas être un «professionnel », etc. De fait, la notion de pair était définie par un ensemble mouvant d'éléments relatifs à la proximité sociale, culturelle, linguistique, religieuse, ethnoraciale. Or, comme l'exprimait une responsable de l'organisation du dépistage : "la spécificité [de ce projet] c'est qu'on prend des gens du cru pour qu'ils fassent comme ils le sentent, par leurs propres initiatives »(Professionnel). Seulement, attribuer au fait « d'être du cru » la capacité de construire et de porter des initiatives perti- 
nentes et effectives pour réaliser ce que les professionnels eux-mêmes ne parviennent pas bien à faire, c'est présupposer que les inégalités sociales face à la prévention peuvent se travailler en améliorant la proximité socioculturelle. Ce présupposé n'est évidemment pas infondé, mais tout se passe comme si cette approche de type « santé communautaire » ne trouvait pas l'assise ou la théorisation qui lui permettrait de s'expliciter clairement dans le cours de cette expérimentation. C'est là un des défis importants pour la pensée des politiques de prévention contemporaine : savoir penser les enjeux des inégalités de santé et des revendications d'égalité et de justice non seulement selon le registre épidémiologique et socio-géo-démographique, mais aussi en faisant place aux expériences sociales, incarnées, situées et historicisées.

\section{Remarques conclusives}

Les programmes de navigateurs représentent manifestement une approche innovante pour développer des politiques de prévention qui tiennent compte non seulement des critères consensuels développés dans les années 1970, mais aussi de la montée des droits individuels et des inégalités sociales de santé. Malgré de nombreuses limites pratiques, politiques et épistémologiques, les médiateurs de santé sont susceptibles de contribuer à enrichir les programmes de santé publique en leur permettant de prendre en considération l'expérience des personnes concernées et possiblement de développer des innovations sur le terrain - à condition qu'on leur en laisse véritablement l'initiative.

L'expérience des navigateurs dans une recherche interventionnelle destinée à changer la participation aux DOCC est révélatrice de l'espace de tensions éthiques dans lequel se développent les programmes de prévention en santé publique. Une première tension vient de la difficulté à intégrer les incertitudes propres à ces programmes de dépistage organisé alors qu'on prend pour norme implicite celle d'un individu rationnel qui, s'il n'en était empêché par différents facteurs psychosociaux, effectuerait normalement ces dépistages. La seconde tension tient à la difficulté de tenir compte de façon globale de la perspective des personnes concernées alors que le dispositif de prévention se déploie dans un processus rationalisé, sectorisé (par pathologie, par types de campagnes de prévention) et orienté par des critères de performance inévitablement trop étroits. La troisième tension se situe à l'articulation entre le déploiement normatif d'un dispositif de prévention biomédicale et le respect de la perspective des personnes et de leur droit à ne pas subir de démarche intrusive au nom 
de leur santé future. La quatrième tension tient à la façon dont on confie à des acteurs minoritaires et peu soutenus la responsabilité de déployer, voire d'inventer, les correctifs commandés par une politique de prévention à la fois universaliste et proportionnée, soucieuse de ne pas renforcer les inégalités sociales de santé.

Ces tensions sont à verser au débat contemporain sur les politiques de santé, et notamment autour de la notion de « culture commune de la prévention ». D'après un rapport de l'Académie de médecine, elle manquerait cruellement ; il faudrait la créer, l'inculquer dès le plus jeune âge (Dreux, 2013). Dans ses propres travaux sur la culture de prévention, Dozon (2001) rappelait que les pratiques de prévention se font suivant des «formes particulières et historiquement attestées » d'un schème universel commun (p. 26). Ce qu'il appelait modèle contractuel, « constitutif de normes, de valeurs, d'habitus [dépassant] largement le souci de soi, mettant en regard une normativité sociale guidant les comportements individuels et des politiques publiques pour les rendre praticables » (p. 42) est traversé par une profonde ambivalence, se manifestant à la fois comme processus de civilisation et comme processus de normalisation. Le dépistage et son lot de technologies, de savoirs, d'éléments de communication, de valeurs, de principes, d'institutions, de territoires, d'acteurs est un bon terrain pour révéler cette ambivalence. L'ethos de la prévention ne se montre pas consensuel, stable et bien fondé sur un petit nombre de principes qui resteraient à appliquer. Il est au contraire dynamique et instable, pris dans une série de tensions qui se révèlent dans des épreuves.

Dès lors, pour contribuer à une éthique en santé publique, il nous faut, non pas d'abord des fondements et des principes intangibles doublés d'une politique d'instruction sanitaire, mais plutôt des moyens d'enquête et d'exploration pluraliste des différentes façons par lesquelles les protagonistes mobilisent des choses qui sont importantes pour eux/elles. De ce point de vue, il nous semble particulièrement fécond pour l'étudier d'associer philosophie morale, enquête qualitative et approche participative. Nous avons besoin, pour mieux comprendre les tenants et aboutissants de la prévention contemporaine, de faire travailler ensemble éthique philosophique et sociologie critique ; mais nous avons également besoin d'apprendre à mieux tenir compte de l'expérience des personnes qui prennent part, de multiples façons (même en ne participant pas) à ces interventions de santé publique. C'est du reste la proposition de Baylis et al. (2008) : puiser dans les travaux féministes concernant les concepts d'autonomie et de justice sociale, pour penser une éthique relationnelle de la santé publique.

Il nous semble que les recherches interventionnelles en santé des populations, en particulier si elles se déploient sur un mode collaboratif, constituent un espace propice à l'exploration des différents registres dans 
lesquels s'incarne la prévention contemporaine. Cependant, il convient de prêter attention à leurs modalités d'évaluation qui, lorsqu'elles sont dominées par les approches médico-économiques et épidémiologiques, orientent implicitement les pratiques, contraignent et limitent l'espace d'apprentissage que constitue pourtant toute recherche-action en réduisant les résultats à l'évolution d'un taux de participation. Il existe un consensus aujourd'hui à propos de l'importance des approches de type « évaluation réaliste » qui ambitionnent de comprendre les processus à travers lesquels une intervention de santé publique produit ses effets, et ce afin de pouvoir valoriser son impact sur les populations cibles à travers un registre plus large de dimensions et permettre de la transférer dans d'autres contextes. Il convient de tirer toutes les conséquences d'une telle ambition, qui gagnerait à être moins réaliste que pragmatiste. Pour ce faire, il faut donner de véritables moyens aux différentes disciplines des sciences humaines et sociales afin de développer et approfondir des enquêtes, non seulement pour décrire les liens de causalité complexes des dispositifs de santé publique, mais aussi pour souligner la dynamique des valeurs en jeu et leurs reconfigurations dans l'étude de leurs conséquences.

\section{Remerciements}

Cette recherche a été soutenue par une subvention de la Ligue contre le Cancer. Elle a bénéficié de l'appui du Centre Hygée, de l'Institut Français de l'Éducation et de l'Université Claude Bernard Lyon1. Nous remercions les relecteurs qui ont permis d'améliorer l'article, Franck Chauvin qui nous a laissé toute marge de manœuvre, Annefleur Perez et Sophie Rousseau pour la mise en place du projet, ainsi que Zahia Adjal, Fouzia Chabani, Ghazi Gharbi, Mokrane Faid et Kevin Vasbinder dont l'inventivité et la ténacité ont beaucoup nourri cette enquête.

Liens d'intérêt : les auteurs déclarent ne pas avoir de lien d'intérêt en rapport avec cet article.

\section{RÉFÉRENCES BIBLIOGRAPHIQUES}

Allary C., Bourmaud A., Tinquaut F., Oriol M., Kalecinski J., Dutertre V., Lechopier N., Pommier M., Benoist Y., Rousseau S., Regnier V., Buthion V., Chauvin F., 2016, ColoNav: patient navigation for colorectal cancer screening in deprived areas - Study protocol, BMC Cancer, 16,1, 416. 
Armstrong N., Eborall H., 2012, The sociology of medical screening: past, present and future, Sociology of Health \& Illness, 34, 161-176.

Barratt A., Mannes P., Irwig L., Trevena L., Craig J., Rychetnik L., 2002, Cancer screening, Journal of Epidemiology and Community Health, 56, 899902.

Baylis F., Kenny N.P., Sherwin S., 2008, A relational account of public health ethics, Public Health Ethics, 1, 3, 196-209.

Borry P., Schotsmans P., Dierickx K., 2005, The birth of the empirical turn in bioethics, Bioethics, 19, 1, 49-71.

Buthion V., Ramone-Louis J., Rousseau S., Chanal M., Amate M., Allary C., 2015, ColoNav : La navigation pour favoriser la participation des populations défavorisées au dépistage du cancer colorectal, Rapport de recherche, Université Lyon2.

Childress J.F., Faden R.R., Gaare R.D., Gostin L.O., Kahn J., Bonnie R.J., Kass N.E., Mastroianni A.C., Moreno J.D., Nieburg P., 2002, Public health ethics: mapping the terrain, The Journal of Law, Medicine \& Ethics, 30, 170-178.

Collectif, 2016, Rapport du Comité d'orientation de la concertation citoyenne et scientifique sur le dépistage du cancer du sein organisée par L'INCa à la demande de la ministre de la Santé.

Dawson A., Verweij M., 2009, Introduction, In : Dawson, A., Verweij, M., eds, Ethics, Prevention, and Public Health, Oxford, Clarendon Press, Oxford University Press, 1-12.

Dewey J., 2011, La formation des valeurs, Paris, La Découverte, Coll. Les empêcheurs de penser en rond.

Dozon J.P., 2001, Quatre modèles de prévention, In : Dozon J.P., Fassin D., eds, Critique de la santé publique : Une approche anthropologique, Paris, Balland, Coll. Voix et Regards. 23-46.

Dreux C. 2013. La culture de prévention en santé : des questions fondamentales, Rapport adopté par l'Académie de médecine le 15 octobre 2013.

Frith L., 2012, Symbiotic empirical ethics: a practical methodology, Bioethics, 26, 4, 198-206.

Gigerenzer G., 2008, Rationality for Mortals. How People Cope with Uncertainty, Oxford, New York, Oxford University Press, Coll. Evolution and Cognition.

Hill C., 2013, Prévention et dépistage des cancers, Bulletin du Cancer, 100, 547-554.

Holland S., 2007, Public Health Ethics, Cambridge, Malden, Polity Press. 
Holme Ø., Bretthauer M., Fretheim A., Odgaard-Jensen J., Hoff G., 2013, Flexible sigmoidoscopy versus faecal occult blood testing for colorectal cancer screening in asymptomatic individuals, Cochrane Database of Systematic Reviews, 9, CD009259.

Juth N., Munthe C., 2012, The Ethics of Screening in Health Care and Medicine, Dordrecht, Springer, Coll. International Library of Ethics, Law and the New Medicine.

Knight R., 2016, Empirical population and public health ethics: A review and critical analysis to advance robust empirical-normative inquiry, Health: An Interdisciplinary Journal for the Social Study of Health, Illness and Medicine, 20, 3, 274-290.

Lang T., 2014, Inégalités sociales de santé, Les Tribunes de la Santé 43, 2, 31-38. Lechopier N., 2015, L'éthique embarquée. Faut-il un éthicien dans une équipe de recherche en santé publique ?, Éthique \& Santé 12, 2, 124-129.

Marmot M., 2005, Social determinants of health inequalities, The Lancet, 365, 1099-1104.

Massé R., 2003, Éthique et santé publique: enjeux, valeurs et normativité, Sainte-Foy, Presses de l'Université Laval, Coll. Sociétés, cultures et santé.

Mazet P., 2013, Analyse compréhensive des motifs de participation et de nonparticipation au dépistage du cancer colorectal des individus vivant dans les territoires précaires, Observatoire des non-recours aux droits et services (ODENORE), Hal-00983467.

Molewijk B., Stiggelbout A.M., Otten W., Dupuis H.M., Kievit J., 2004, Empirical data and moral theory. A plea for integrated empirical ethics, Medicine, Health Care and Philosophy, 7, 1, 55-69.

Parker V.A., Clark J.A., Leyson J., Calhoun E., Carroll J.K., Freund K.M., Battaglia T.A., 2010, Patient navigation: development of a protocol for describing what navigators do, Health Services Research, 45, 2, 514-531.

Pommier M., 2015, Le projet Colonav à l'épreuve du terrain, Rapport de recherche, Institut Français de l'Éducation, École Normale Supérieure de Lyon.

Ponchon T., 2009, Complications de la coloscopie : synthèse des connaissances en vue de l'évaluation du dépistage organisé, Bulletin Épidémiologique Hebdomadaire, 2-3, 14-16.

Potter M.B., 2013, Strategies and resources to address colorectal cancer screening rates and disparities in the United States and globally, Annual Review of Public Health, 34, 1, 413-429.

Putnam H., 2004, Fait/valeur : la fin d'un dogme, et autres essais, Paris, Éditions de l'Éclat. 
Skrabanek P., 1990, Why is preventive medicine exempted from ethical constraints? Journal of Medical Ethics, 16, 4, 187-190.

Steckelberg A., Hulfenhaus C., Haastert B., Muhlhauser I., 2011, Effect of evidence based risk information on "informed choice" in colorectal cancer screening: randomized controlled trial, British Medical Journal, 342, d3193. Upshur R.E., 2002, Principles for the justification of public health intervention, Canadian Journal of Public Health/Revue Canadienne de Santé Publique, 93, 2, 101-103.

Wilson J., Jungner G., 1970, Principes et pratique du dépistage des maladies, Genève, OMS, Coll. Cahiers de santé publique, 34. 
ABSTRACT

\section{Supporting and preventing. \\ Ethical tensions within a colorectal cancer screening campaign}

This paper presents the results of an inquiry in prevention ethics, embedded in a population health intervention research. "Navigators " were key players deployed with the final aim of rectifying unequal participation in colorectal cancer screening campaign in 5 French departments. Four ethical tensions emerged during field work regarding (1) uncertainties in the test itself, (2) organization of the screening campaign, (3) intrusiveness and privacy, (4) proportionate universalism. These tensions are described here using a pragmatist approach. Attention was paid to hardships as experienced by a range of prevention actors. Investigation on facts and reflection on values were carried out in a same movement.

\section{RESUMEN}

\section{Acompañar y prevenir. Tensiones éticas en la detección del cancer colorrectal}

Este artículo presenta los resultados de una encuesta sobre la ética de la prevención. Se llevó a cabo en una investigación intervencional implicando mediadores (navegadores) para corregir las desigualdades de participación en la campaña de detección del cáncer colorrectal. Cuatro tensiones éticas fueron descubiertas: sobre la incertidumbre del test, la organización de la campaña, la intromisión en la esfera privada y el universalismo proporcional. Estas tensiones se describen en una investigación basada en un enfoque pragmático, atento a las dificultades que enfrentan los actores de la prevención, integrando en un mismo movimiento la investigación sobre los hechos y la reflexión sobre los valores. 